\title{
Switching from Endoscopic Extraperitoneal Radical Prostatectomy to Robot-Assisted Laparoscopic Prostatectomy: Comparing Outcomes and Complications
}

\author{
Robert Wagenhoffer ${ }^{a}$ b Maren Gruner ${ }^{a}$ Jan Schymik ${ }^{c}$ Lydia Schachtner $^{a}$ \\ Liviu Neagoe $^{a}$ Christine Berg $^{b}$ Andreas Schlichter ${ }^{b}$ Andreas Manseck $^{a}$ \\ ${ }^{a}$ Department of Urology, Klinikum Ingolstadt, Ingolstadt, ${ }^{\mathrm{b}}$ Department of Urology, Wald-Klinikum Gera, Gera, and \\ 'University of Munich, Munich, Germany
}

\section{Key Words}

Prostate cancer · Prostatectomy · Endoscopic

extraperitoneal radical prostatectomy $\cdot$ Robot-assisted

laparoscopic prostatectomy

\begin{abstract}
Objective: Endoscopic extraperitoneal radical prostatectomy (EERPE) and robot-assisted laparoscopic prostatectomy (RALP) are minimally invasive surgical techniques to treat localized prostate cancer. We report the outcome and complications of these two techniques conducted by one individual surgeon. Patients and Methods: 86 patients underwent EERPE between January 2008 and June 2011, and 100 patients underwent RALP between August 2011 and October 2012. All surgeries were performed by one single surgeon. Results: The patients of the EERPE and RALP groups had similar clinical characteristics in PSA, prostate volume and D'Amico classification, and were significantly different in their age and BMI as well as in the number of prior surgeries. RALP surgeries were significantly slower (183 vs. $157 \mathrm{~min}$ ) but also involved lower blood loss (147 vs. $245 \mathrm{ml}$ ). Pathological stages and positive surgical margins were similar in both groups. Complications were assessed by the ClavienDindo classification. 6 patients in the EERPE group and 3 patients of the RALP group suffered major complications (Illb-
\end{abstract}

(c) 2015 S. Karger AG, Basel

0042-1138/15/0954-0380\$39.50/0
IV). Conclusion: Altogether our results indicate that the learning curve for RALP was short after experience with EERPE. We hypothesize that this is more a result of the surgical experience of the surgeon with the EERPE than on the robotic technique.

(c) 2015 S. Karger AG, Basel

\section{Introduction}

Endoscopic extraperitoneal radical prostatectomy (EERPE) and robot-assisted laparoscopic prostatectomy (RALP) are minimally invasive surgical techniques to treat localized prostatic cancer. Before EERPE was established, laparoscopic radical prostatectomy (LRP), a transperitoneal technique introduced by Schuessler et al. [1] in 1997, had been the primary laparoscopic technique. LRP became more established at the end of the 1990s in some centers in Europe [2-5]. Although LRP led to good oncological and functional results, the technique did not spread widely. The main reasons were long surgery time in addition to a long learning curve. The extraperitoneal technique was extensively used and standardized by Stolzenburg et al. [6,7]. They reported the advantage of EERPE: minimal invasive surgery combined with an extraperitoneal approach as within open retropubic prostatectomy.

Dr. Robert Wagenhoffer

Department of Urology, Klinikum Ingolstadt

Krumenauerstrasse 25

DE-85049 Ingolstadt (Germany)

E-Mail robertwagenhoffer@yahoo.de 
Table 1. Baseline characteristics

\begin{tabular}{lccc}
\hline & EERPE & RALP & $\mathrm{p}$ \\
\hline Number of patients & 86 & 100 & 0.017 \\
Age, years & $65.8(46-78)$ & $63.6(48-75)$ & 0.116 \\
PSA, ng/ml & $7.2(0.2-50.0)$ & $8.8(1.4-75)$ & 0.103 \\
Prostate avolume, ml & $37.6(15-120)$ & $41.0(16-142)$ & 0.003 \\
BMI & $27.2(20.4-42.1)$ & $28.7(15.7-46.5)$ & 0.787 \\
D'Amico score & & & 0.830 \\
$\quad$ Low & $30(34.9)$ & $33(33)$ & 0.602 \\
$\quad$ Intermediate & $35(39.5)$ & $38(38)$ & 0.512 \\
$\quad$ High & $21(25.6)$ & $29(29)$ & 0.004 \\
Prior surgery (one) & $18(20.9)$ & $25(25)$ & 0.015 \\
Prior surgeries (two or more) & $6(7.0)$ & $22(22)$ & 0.116 \\
Inguinal hernia repair (open, TAPP) & $9(10.5)$ & $24(24)$ & 0.509 \\
Appendectomy (open, laparoscopic) & $12(14.0)$ & $23(23)$ & $>0.999$ \\
Cholecystectomy (open, laparoscopic) & $3(3.5)$ & $6(6)$ & 0.125 \\
Colonresection (open, laparoscopic) & $1(1.2)$ & $1(1)$ & 0.025 \\
Transurethral prostate resection & $1(1.2)$ & $6(6)$ & $2(2)$ \\
Hormone therapy & $9(10.5)$ & & \\
\hline
\end{tabular}

Values are presented as $\mathrm{n}(\%)$ or means (range).

The first RALP was performed in 2000 by Binder and Kramer [8] in Frankfurt, Germany. However, it was first established in the US [9-11]. The advantages of the robotic technique over the standard EERPE are the 3-dimensional view (as in an open surgical technique), tenfold magnification, $7^{\circ}$ of movement freedom of the instruments instead of $3^{\circ}$ as with standard laparoscopy, motion scaling and a more ergonomic position for the surgeon. As a result of these advantages, the learning curve can be reduced [12] compared to the standard LRP. The aim of this paper is to analyze the results from both surgical techniques - EERPE and RALP - conducted by one individual surgeon who switched from EERPE to the RALP technique in order to provide some evidence on differences in outcome and complications.

\section{Patients and Methods}

We investigated a sample of 186 patients that underwent a prostatectomy by one individual surgeon (R.W.). 86 patients underwent EERPE between January 2008 and June 2011. After June 2011, the surgeon switched the surgery method from EERPE to RALP. Afterwards, 100 patients underwent RALP between August 2011 and October 2012. This clear switch of the surgery method over time allows us to identify the effect of the surgical technique on the outcome without having to consider patient inclusion to either method. Table 1 summarizes the baseline characteristics of each group. EERPE patients were significantly older than RALP patients (65.8 vs. 63.6 years). Furthermore, the BMIs were significantly different between both groups: 27.2 in the EERPE versus 28.7 in the RALP group. An important significant difference was the number of previous abdominal surgeries. RALP patients underwent significantly more prior surgeries. Only 24 patients in the EERPE group underwent prior surgeries while 47 RALP patients had one or more of those prior surgeries. The mean PSA was 7.2 $\mathrm{ng} / \mathrm{ml}(0.2-50.0)$ in the EERPE group versus $8.8 \mathrm{ng} / \mathrm{ml}(1.4-75)$ in the RALP group, and the mean prostate volume was $37.6 \mathrm{ml}$ (15$120)$ versus $41.4 \mathrm{ml}(16-142)$.

EERPE was performed as described by Stolzenburg et al. [13]. RALP was performed with the 4-arm standard S system (Intuitive Surgical ${ }^{\circledR}$ ) using the 6-port transperitoneal technique described by Menon et al. [14].

\section{Statistical Analysis}

For continuous data, we report means and the smallest, respectively largest value in parentheses. Categorical variables are presented in absolute and relative (in parentheses) occurrences. Continuous variables are compared by Mann-Whitney U test statistics. For categorical variables, $\chi^{2}$ test statistics are calculated when there are at least five occurrences within a category. When there are less than five occurrences within one category, Fisher's exact test statistics are used. Generally, $\mathrm{p} \leq 0.05$ is considered as statistically significant; all $\mathrm{p}$ values correspond to 2 -sided test statistics. In table 2 , the effects of the RALP method on blood loss, continence and surgical margins are denoted in bold. Estimates in column 1 correspond to ordinary least squares coefficients and hence may be interpreted as difference in blood loss during surgery (in ml). Estimates in columns 2 and 3 were obtained from logistic regressions and report odds ratios. All calculations were performed in STATA 12.1 (StataCorp LP, College Station, Tex., USA). 
Table 2. Blood loss, continence and positive surgical margins

\begin{tabular}{llll}
\hline & $\begin{array}{l}\text { Blood loss }(\mathrm{ml}), \\
\text { multivariate analysis }\end{array}$ & $\begin{array}{l}\text { Continence (6 months), } \\
\text { logistic analysis }\end{array}$ & $\begin{array}{l}\text { Complete resection, } \\
\text { logistic analysis }\end{array}$ \\
\hline RALP & $\mathbf{- 8 4 . 1 3 ^ { * * * }}$ & $\mathbf{1 . 7 7 4}$ & $\mathbf{0 . 9 7 7}$ \\
Age (10 years) & $29.3^{* *}$ & 1.004 & 1.002 \\
PSA (10 ng/ml) & -2.2 & 1.008 & 1.002 \\
Prostate volume (10 ml) & -1.5 & 0.999 & 1.001 \\
BMI (10 points) & 4.7 & 0.998 & 1.000 \\
Prior surgery (one) & $-37.23^{* *}$ & 0.82 & 0.587 \\
Prior surgeries (two or more) & $-42.11^{* *}$ & 0.585 & 4.713 \\
High D'Amico score & -6.29 & 1.121 & $0.163^{* *}$ \\
Intermediate D'Amico score & 21.46 & 0.910 & 0.616 \\
\hline
\end{tabular}

This table reports multivariate regression results regarding blood loss (column 1), continence (column 2) and complete resection (column 3) to control for significant baseline characteristics. Numbers in column 1 correspond to marginal effects on blood loss in ml. Numbers in columns 2 and 3 report odds ratios (relative risk ratios). ${ }^{* *} \mathrm{p}<0.05 ;{ }^{* * *} \mathrm{p}<0.001$. See text for additional explanations.

Table 3. Perioperative data

\begin{tabular}{lccr}
\hline & EERPE & RALP & p \\
\hline Surgery time, min & $157(85-252)$ & $183(118-360)$ & $<0.001$ \\
Blood loss, ml & $245(15-1,000)$ & $147(20-900)$ & $<0.001$ \\
Transfusion & $0(0)$ & $0(0)$ & $>0.999$ \\
Conversion & $1(1.2)$ & $0(0)$ & 0.462 \\
Lymphadenectomy & $82(95.3)$ & $100(100)$ & 0.029 \\
Nerve-sparing surgery & $17(19.8)$ & $45(45)$ & $<0.001$ \\
Umbilical hernia repair & $0(0)$ & $4(4)$ & 0.125 \\
TAPP & $0(0)$ & $6(6)$ & 0.031 \\
Pathological stage & & & \\
$\quad$ pT2a & $11(12.8)$ & $8(8)$ & 0.282 \\
pT2b & $2(2.3)$ & $3(3)$ & $>0.999$ \\
pT2c & $55(64)$ & $61(61)$ & 0.334 \\
pT3a & $9(10.5)$ & $22(22)$ & 0.016 \\
pT3b & $9(10.5)$ & $6(6)$ & 0.561 \\
Positive surgical margins & $11(12.8)$ & $12(12)$ & 0.706 \\
$\quad$ pT2R1 & $4(5.9)$ & $9(4.2)$ & 0.835 \\
pT3R1 & $7(38.9)$ & $7.2(5-63)$ & $<0.001$ \\
Mean catheter time, days & $9.2(5-50)$ & $91(91)$ & 0.405 \\
Continence after 6 months & $75(87)$ &
\end{tabular}

Values are presented as n (\%) or means (range).

\section{Results}

Table 3 lists perioperative data. EERPE surgeries were performed significantly faster relative to RALP. Average surgery time was 157 min (85-252) in the EERPE group versus 183 min (118-360) in the RALP group. However, a significantly lower blood loss occurred during RALP sur- geries: $245 \mathrm{ml}(15-1,000)$ in the EERPE versus $147 \mathrm{ml}(20-$ $900)$ in the RALP group. We also conducted a multivariate regression analysis where we controlled for age, PSA, BMI, prostate volume, prior surgeries and D'Amico classification to isolate and quantify the effect of the surgery method on the blood loss during surgery. We find here that the RALP method leads to an approximate $84-\mathrm{ml}$ lower blood 
Table 4. Complications

\begin{tabular}{|c|c|c|c|c|}
\hline & Clavien grade & EERPE, $\mathrm{n}$ & RALP, n & $\mathrm{p}$ \\
\hline \multicolumn{5}{|l|}{ Intraoperative complications } \\
\hline Rectal injury & IIIb & 2 & 0 & 0.212 \\
\hline \multicolumn{5}{|l|}{ Early complications $<1$ month postoperatively } \\
\hline Urinary retention & I & 1 & 1 & $>0.999$ \\
\hline Renal insufficiency (excluding dialysis) & I & 0 & 1 & $>0.999$ \\
\hline Prolonged catheterization $>14$ days & Id & 7 & 3 & 0.191 \\
\hline Ureter injury & IIIb & 2 & 0 & 0.212 \\
\hline Symptomatic hydronephrosis & IIIb & 0 & 1 & $>0.999$ \\
\hline Anuria & IIIb & 1 & 0 & 0.462 \\
\hline Bowel injury & IIIb & 0 & 1 & $>0.999$ \\
\hline Rectourethral fistula & IIIb & 1 & 0 & 0.462 \\
\hline Renal insufficiency (including dialysis) & IVa & 0 & 1 & $>0.999$ \\
\hline \multicolumn{5}{|l|}{ Late complications $>1$ month postoperatively } \\
\hline Symptomatic lymphocele & IIIa & 0 & 3 & 0.250 \\
\hline Total & & $14(16.3 \%)$ & $11(11 \%)$ & 0.293 \\
\hline
\end{tabular}

loss. Because no perioperative blood transfusion was needed in either group, this difference is statistically but not clinically significant. One EERPE patient needed conversion to open surgery. Lymphadenectomy was performed in $95.3 \%$ of the EERPE patients and $100 \%$ of the RALP patients. Nerve-sparing surgery was indicated in preoperatively potent patients (evaluated with an IIEF-5 questionnaire) with Gleason score $\leq 7 \mathrm{a}$, PSA $<10 \mathrm{ng} / \mathrm{ml},<50 \%$ tumor in biopsy and no palpable tumor on the ipsilateral side. Nerve-sparing surgery (uni- or bilateral) was performed on $19.8 \%$ of the EERPE patients and on $45 \%$ of the RALP patients. 4 RALP patients additionally underwent an umbilical hernia repair and 6 RALP patients underwent transabdominal preperitoneal plastic with mesh (TAPP).

The distribution of pathological stages was similar for both groups. $12 \%$ of the RALP patients had positive surgical margins. Within these patients, $4.2 \%$ had a pathological stage of pT2 and $32.1 \%$ of pT3. In the EERPE group, $12.8 \%$ of the patients had positive surgical margins, and $5.9 \%$ of these had a pathological stage of pT2 and $38.9 \%$ of pT3. The odds ratio of 0.977 in logistic regression (table 2, column 3 ) indicates that there is also no significant difference regarding positive surgical margins between both methods after controlling for the major baseline characteristics.

The mean catheter time was significantly shorter in the RALP group. Average catheter time was 9.2 days in the EERPE group versus 7.2 days in the RALP group. Continence was evaluated with the International Consultation on Incontinence Questionnaire - Urinary Incontinence
Short Form (ICIQ-UI Short Form) with an additional question concerning the number of pads. After 6 months, continence was very similar for both groups. $87 \%$ of the EERPE patients and $91 \%$ of the RALP patients were continent (0 or 1 security pad). Also here, we found no significant difference in the logistic regression (table 2, column 2).

Complications are listed in table 4 , and are graded using the Clavien-Dindo classification [15]. The total complication rate was $16.3 \%$ in the EERPE group and $11 \%$ in the RALP group. Major complications (Clavien grade IIIb-IV) occurred in 6 EERPE patients. Major complications in the RALP group occurred in 3 cases.

\section{Discussion}

EERPE and RALP are widely used minimally invasive surgical techniques to treat localized prostatic cancer. To compare these two techniques, we reported perioperative data as well as intraoperative, early and late stage complications of 186 cases, all conducted by one individual surgeon. It must be emphasized that the surgeon switched from the EERPE to RALP over time which allows us to draw two main conclusions. First, our study may be interpreted as a case study that documents the switch of one surgeon from EERPE to RALP. We document that this switch from EERPE to RALP can be carried out without major difficulties. Consequently, we conclude that the RALP learning curve was short in this case. Second, since the switch of technique occurred over time, our study al- 
lows us to identify the effect of the surgery method on outcome and complications without having to worry about patient inclusion to one of both methods. Here, we find that both methods lead to comparable outcomes.

In this study, the mean operating time was $157 \mathrm{~min}$ in the EERPE group and $183 \mathrm{~min}$ in the RALP group. The longer mean operating time of our RALP group could be explained by a significantly higher number of nerve-sparing surgeries. 4 RALP patients additionally underwent an umbilical hernia repair and 6 RALP patients underwent a TAPP.

The mean blood loss was 245 versus $147 \mathrm{ml}$. There was no need for perioperative blood transfusions. Stolzenburg et al. [13] had after 300 EERPEs (178 patients undergoing lymphadenectomy) a mean operating time of $140 \mathrm{~min}$, and 4 patients (1.3\%) needed a blood transfusion. Rocco et al. [16] showed after 120 RALPs a mean operating time of $215 \mathrm{~min}(165-450)$ and a mean blood loss about $200 \mathrm{ml}(50-2,000)$.

The critical criterion to evaluate an oncosurgical technique is local cancer control. The positive margin is a risk factor for disease recurrence. In this study the R1 rates correspond with the numbers published in other centers. Stolzenburg et al. [17] published after 1,300 EERPEs a R1 rate of $9.8 \%$ for stage pT 2 and $34.3 \%$ for stage pT3. Patel et al. [18] showed after 500 RALPs a total R1 rate of 9.4\%; in the first 100 RALPs it was $13 \%$. Padavano et al. [19] published after 153 RALPs, R1 rates of $30 \%$ at stage pT2 and $67 \%$ at stage pT3.

The total complication rate in our study was $16.3 \%$ in the EERPE group and $11 \%$ in the RALP group. This is in accordance with analyses of other centers. In the multianalysis review from Coelho et al. [20] containing only studies with more than 250 patients, the complication rate for RALP was about $10.3 \%$ (4.3-15.7). Hu et al. [21] published in a serial of 358 LRPs and 322 RALPs 9 rectal injuries (Clavien grade IIIb), and in 5 patients a rectourethral fistula occurred. All rectal injuries happened in the LRP group. A ureter injury occurred (Clavien grade IIIb) in 1 patient in the LRP group and in 1 patient in the RALP group. Murphy et al. [22] described a total complication rate of $15.75 \%$ after 400 RALPs; 21 patients had a Clavien III complication. Among these were 5 patients with a rectal injury. Bhandari et al. [23] assessed after the analysis of two subsequent groups $(\mathrm{n}=200)$ undergoing RALP that the complication rate did not decrease according to the experience of the surgeon. He even had 2 bowel injuries in the second group. We conclude that our outcomes are comparable to the elite center outcomes presented in the literature.
In our study, the surgeon was experienced in laparoscopy before learning the robotic technique. Our results indicate that in this case, the learning curve for RALP was very short. The parameters to rate the learning curve include operating time, blood loss, need for transfusions, occurrence of perioperative complications, and oncological and functional results. A major advantage of RALP should be a short learning curve for an inexperienced surgeon in laparoscopy. Patel et al. [10] published that after 200 initial surgeries there was a mean operating time of $141 \mathrm{~min}$ and the learning curve involved 20-25 surgeries. Ahlering et al. [12] postulated that an inexperienced surgeon in laparoscopy with good open surgery skills can gain after 8-12 RALPs very similar results as an experienced laparoscopy surgeon after more than 100 LRPs. However, Doumerc et al. [24] shows with a higher number of patients that there is a much longer learning curve for RALP. In their analysis, a very skilled open surgeon $(>2,000$ retropubic radical prostatectomies) who was inexperienced in laparoscopy needed 110 surgeries to get to a mean operating time of $3 \mathrm{~h}$. The number of positive margins was stable after 120 RALPs. There is a controversial discussion of the role of laparoscopic experience needed for RALP. Experience in laparoscopy is definitely not coactively needed, but it facilitates the change to the robotic techniques enormously. In the authors' opinion, it is not possible to draw a general conclusion how many surgeries are necessary to reach the end of the learning curve. In general, the learning curve is influenced by the surgical skills of the surgeon, patient characteristics, surgery frequency and experience of the center with the described surgical technique.

\section{Conclusions}

The outcomes, measured by positive surgical margins, severe complications and continence, were generally similar under both techniques. We also find significant difference in average surgery time and mean catheter time. The blood loss was statistically, but not clinically, significant. Our results indicate that switching from EERPE allowed good results by RALP to be achieved in very short time.

\section{Disclosure Statement}

The authors report no conflicts of interest.
Wagenhoffer et al. 


\section{References}

1 Schuessler WW, Schulam PG, Clayman RV, Kavoussi LR: Laparoscopic radical prostatectomy: initial short-term experience. Urology 1997;50:854-857.

2 Guillonneau B, Vallencien G: Laparoscopic radical prostatectomy: the Montsouris experience. J Urol 2000;163:418-422.

3 Rassweiler J, Sentker L, Seemann O, Hatzinger M, Rumpelt HJ: Laparoscopic radical prostatectomy with the Heilbronn technique: an analysis of the first 180 cases. J Urol 2001; 166:2101-2108.

4 Anastasiadis AG, Salomon L, Katz R, Hoznek A, Chopin D, Abbou C: Radical retropubic versus laparoscopic prostatectomy a prospective comparison of functional outcome. Urology 2003;62:292-297.

5 Türk I, Deger IS, Winkelmann B, Roigas J, Schönberger B, Loening SA: Die laparoskopische radikale Prostatektomie. Erfahrungen mit 145 Eingriffen. Urologe A 2001;40:199_ 206.

6 Stolzenburg JU, Do M, Rabenalt R, et al: Endoscopic extraperitoneal radical prostatectomy: initial experience after 70 procedures. J Urol 2003;169:2066-2071.

7 Stolzenburg JU, Rabenalt R, Do M, et al: Endoscopic extraperitoneal radical prostatectomy: oncological and functional results after 700 procedures. J Urol 2005; 174:1271-1275.

8 Binder J, Kramer W: Robotically-assisted laparoscopic radical prostatectomy. BJU Int 2001;87:408-410.
9 Tewari A, Srivasatava A, Menon M; Members of the VIP Team: A prospective comparison of radical retropubic and robot-assisted prostatectomy: experience in one institution. BJU Int 2003;92:205-210.

10 Patel VR, Tully AS, Holmes R, Lindsay J: Robotic radical prostatectomy in the community setting - the learning curve and beyond: initial 200 cases. J Urol 2005;174:269-272.

11 Van Appledorn S, Bouchier-Haeys D, Agarwal D, Costello AJ: Robotic laparoscopic radical prostatectomy: setup and procedural techniques after 150 cases. Urology 2006;67: 364-367.

12 Ahlering TE, Skarecky D, Lee D, Clayman RV: Successful transfer of open surgical skills to a laparoscopic environment using a robotic interface: initial experience with laparoscopic radical prostatectomy. J Urol 2003;170: 1738-1741.

13 Stolzenburg JU, Truss MC, Rabenalt R, et al: Die endoskopische extraperitoneale radikale Prostatektomie (EERPE). Ergebnisse nach 300 Eingriffen. Urologe A 2004;43:698-707.

14 Menon M, Shrivastava A, Kaul S, et al: Vattikuti Institute prostatectomy: contemporary technique and analysis of results. Eur Urol 2007;51:648-658.

15 Dindo D, Demartines N, Clavien PA: Classification of surgical complications: a new proposal with evaluation in a cohort of 6336 patients and results of a survey. Ann Surg 2004; 240:205-213.

16 Rocco B, Matei DV, Melegari S, et al: Robotic vs. open prostatectomy in a laparoscopically naïve centre: a matched pair analysis. BJU Int 2009; 104:991-995.
17 Stolzenburg JU, Rabenalt R, Do M, et al: Endoscopic extraperitoneal radical prostatectomy: the University of Leipzig experience of 1300 cases. World J Urol 2007;25:45-51.

18 Patel VR, Thaly R, Shah K: Robotic radical prostatectomy: outcomes of 500 cases. BJU Int 2007;99:1109-1112.

19 Padavano J, Schaffer L, Fannin E, et al: Robotic radical prostatectomy at a teaching community hospital: outcomes and safety. JSLS 2011;15:193-199.

20 Coelho RF, Rocco B, Patel MB, et al: Retropubic, laparoscopic and robot-assisted radical prostatectomy: a critical review of outcomes reported by high-volume centers. J Endourol 2010;24:2003-2015.

$21 \mathrm{Hu}$ JC, Nelson RA, Wilson TG, et al: Perioperative complications of laparoscopic and robotic assisted laparoscopic radical prostatectomy. J Urol 2006;175:541-546.

22 Murphy DG, Kerger M, Crowe H, Peters JS Costello AJ: Operative details and oncological and functional outcome of robotic-assisted laparoscopic radical prostatectomy: 400 cases with a minimum of 12 months follow-up. Eur Urol 2009;55:1358-1367.

23 Bhandari A, McIntire L, Kaul SA, Hemal AK Peabody JO, Menon M: Perioperative complications of robotic radical prostatectomy after the learning curve. J Urol 2005; 174:915-918.

24 Doumerc N, Yuen C, Savdie R, et al: Should experienced open prostatic surgeons convert to robotic surgery? The real learning curve for one surgeon over 3 years. BJU Int 2010;106: 378-384. 